Eavourable a result even, as in Mr. Curling's patient, may ensue; but we are very much afriid, in the event of recovery from the operation, that a recurrence, sooner or later, of the disease will manifest itself. As it was known for sume days beforehand that this procedure would be attempted, a good deal of interest in consequence was excited in the surgical world, and a large number of gentlemen, including many of the most distinguished surgeons in the metropolis, were present, the theatre being literally crammed. This operation is by no means a eommon one in civil practice, nor is it often seen in our London hospitals; yet the present instance forms the third performed within twelve months. As it was done under the influence of chloroform, the utmost quiet was shown by the patient, who never moved the whole time of its performance, which was necessarily tedious from the extension of the disease through ont the thigh. Those present, therefore, who expected to see the brilliant operation performed by transfixion, as described in our surgical works, must have been disappointed, as that metrod cannot be attempted under the conditions in which this patient laboured.

The patient was a healthy man, aged fifty-two, who sustained a simple fracture of the upper third of the right femur three years ago, and was treated in this hospital by Mr. Lawrence. Nothing unusual occurred in the healing of the fracture; he was discharged cured from the hospital, and walked about quite well 3 to nine months ago, when a swelling commenced at the apper part of the same thigh, accompanied with an inability of cither using the limb, or supporting the weight of the body upon it; the swelling gradually increased up to the present time, and now occupies fully one half of the thigh, which is misshapen, and prominent from its appearance. The most careful examination satisfied Mr. Stanley that the disease originated in the femur, and most probably at the seat of the fractare, but not beyond it. There was no doubt as to its malignancy, and the only question to be considered was the propriety of removal at the hip-joint, which was determined upon by Mr. Stanley and his colleagues, who agreed unanimously in the propriety of performing it, as affording, in fact, the only chance for the poor man's life. Chloroform was accordingly given on the 28 th of March, when Mr. Stanley proeded to remove the limb, Mr. Paget having command of the femoral artery at the groin, whilst Mr. Wormald had charge of the limb. An incision was made from within outwards, thus cufting the anterior flap first, across the upper part of the tomour, which from its invasion, or, rather, encroachment upon strounding parts, necessitated slow and careful dissection; the femoral vessels were then tied. The capsular ligament was now divided, and the head of the bone disarticulated during rotation of the limb, downwards and outwards, by Mr. Wormald. The posterior flap-very much longer than the anterior-was then completed, by cutting more deeply from within outwards, and the limb was removed, the operation from first to last occupying fifteen minutes. A number of small vessels were now tied, the edges of the flaps were brought together by sutures, and the stump firmly strapped with wide bands of adhesive plaster. The patient was then removed to the wards. Very little blood was lost throughout the operawion.

The limb was then examined, a transverse section being made through the middle of the tumour with a knife and saw, and a zsection through the head and neck of the thigh-bone. The tumour turned out to be medullary cancer, of moderately firm consistence, growing around the femur, and infiltrating the interior of the bone, in all probability commencing within the bone, and extending around it. The section of the head and mecle of the bone showed their interior completely filled with soft whitish medullary matter similar to that around the shaft, and extending downwards through the trochanter major. The capsular ligament and cartilages of the head of the bone were werfeouly free from disease; they had not become penetrated by the eancer, fortunately for the patient. In some remarks made afterwards by Mr. Stanley, when examining this part of the bone, he observed that had they been aware that the disease exterded so high up, as was here shown, they might have paused before doing the operation; still under all the circum stances, he thought it as well the limb had been taken off as the cancer did not extend either beyond or into the joint, and the object of its performance was accomplished, by the complete removal of the disease. No other parts of his body were found to be diseased, on the most careful examination by Dr. Baly and others, which could militate against operative proceedings, and on considering all the circumstances of the case, they were zot unfavourable in leading him to expect a satisfactory result fhe operation. He remarked that the slow progress of the operation was a necessary consequence of the spread of the disease, and the femoral artery had to be tied immediately below Poupart's ligament.

We shall watch the progress of the patient while in the hospital, and give the result at a later date.

\section{ST. PANCRAS ROYAL DISPENSARY.}

CONGENITAL EXTROVERSION OF THE BLADDER IN AN INFANT; INCURABLE NATURE OF THE AFFECTION.

(Under the care of Dr. GrBb.)

Ir is truly remarkable what strange and singular deformities occasionally present themselves to the notice of both the physician and the surgeon at our various public charities. Some of these are common enough, whilst others again are rarely to be met with. Of the latter class, we may include the peculiar and incurable congenital malformation known as extroversion of the bladder. It has been our province to have seen this affection as many as three or four times; on one occasion in a patient, otherwise in good health, who had attained to adult age. The deformity consists of an absence of the anterior wall of the bladder, with a deficiency in the corresponding part of the abdominal parietes. The posterior wall of the bladder is pushed forwards by the pressure of the bowels, and a tumour is consequently formed, which projects just above the pubes, and varies in size from a peach to that of an orange. The surface of this tumour is red, vascular, and papillated, evidently composed of inucous membrane; and at its lower part may generally be seer the orifices of the ureters, which either discharge the urine in drops or in a stream. A case of this kind we recently saw at this dispensary, which was first examined by Dr. Gibb on the 2Sth of February, the little patient being an infant born at the full period on the 25th of February, whose mother, aged forty-two, was a lying-in patient of the dispensary. This was her third child. Nothing unusual occurred at birth, but on examining to ascertain the sex of the child, the presence of something strange was determined. The condition of things already described as generally met with in similar cases was present; the tumour was very prominent, round, and globular, the size of a peach, the mucous membrane being of a blood-red, shiny colour, with the papilla very distinetly seen. The orifices of the ureters projected forwards, at the lower aspect of the bladder, the size of peas, from which flowed urine drop by drop. The penis projected forward from the sides of the pubes, and was irregularly developed, the glans being divided into two portions; the urethra was an open canal running along its dorsum, and a fold of foreskin was situated beneath the glans. The testicles had descended into the scrotum. There was some cutaneous inflammation between the navel and margins of the tumour, which afterwards disappeared; and in the course of some days the prominence of the tumour became sensibly diminished, somewhat contracted, and assumed a deep pink colour, instead of the biood-red. Drops of urine still flowed as usual, occasionally in small streams when crying, and the general health was perfect. No donbt the nreters will become dilated into pouches after a while, and thus form reservoirs for the urine.

We subsequently saw the child, at University College Hospital, on the 25th of March, when it was shown to the pupils; the nature of the affection was mentioned by Mr. Erichsen, and he spoke of the incurable nature of the deformity. $\mathrm{He}$ described a good example of it in 1845, with a full description of the mechanism of the passage of the urine in similar instances.

We have no donbt many surgeons would not hesitate to attempt an operation for relief here, but it is at best a hazardous procedure, although we believe cases have turned out well in America.

Should this child grow up, a properly-devised instrument can be worn to receive and collect the urine. This consists of a hollow shield strapped over the part, communicating, by means of tube, with an india-rubber bottle, that may be attached along the inside of the thigh.

\section{CIINICAL RECORDS.}

ISCHURIA RENALIS, FOR FIFTY-STX HOURS, AFTER PERINEAL SECTION,

WHEN describing, a fortnight ago (at page 317 ), the performance of perineal section upon a man, by Mr. Erichsen, with Mr. Marshall's new instruments, we mentioned that the vapour of amylene was used instead of chloroform, as the latter had 